\title{
Urinary Metabolites of Nobiletin Orally Administered to Rats
}

\author{
Takaaki Yasuda,${ }^{a}$ Yukako Yoshimura, ${ }^{a}$ Hisako Yabuki, ${ }^{a}$ Takahiro Nakazawa, ${ }^{a}$ Keisuke Ohsawa, ${ }^{*, a}$ \\ Yoshihiro MIMAKI, ${ }^{b}$ and Yutaka SASHIDA ${ }^{b}$ \\ ${ }^{a}$ Department of Phytochemistry, Tohoku Pharmaceutical University; 4-4-1 Komatsushima, Aoba-ku, Sendai, Miyagi \\ 981-8558, Japan: and ${ }^{b}$ Laboratory of Medicinal Plant Science, School of Pharmacy, Tokyo University of Pharmacy and \\ Life Science; 1432-1 Horinouchi, Hachioji, Tokyo 192-0392, Japan. $\quad$ Received June 24, 2003; accepted August 27, 2003
}

\begin{abstract}
During the course of our systematic investigation of the metabolism of flavonoids, the polymethoxyflavone nobiletin, occurring in the fruits of Citrus depressa, was orally administered to rats. The urinary metabolites were separated and identified by three-dimensional HPLC equipped with a photodiode array detector and the structure was determined by spectroscopic methods to be $4^{\prime}$-hydroxy-3',5,6,7,8-pentamethoxyflavone (1).
\end{abstract}

Key words nobiletin; metabolism; Citrus depressa; polymethoxyflavone; demethylation

Nobiletin is a polymethoxylated flavone, which is specifically found in the fruits of Citrus depressa, probably as a natural resistance factor against pathogenic fungi. ${ }^{1)}$ Recently, several biological activities of nobiletin have been observed, including the ability to induce differentiation of mouse myeloid leukemia cells, ${ }^{2}$ to exhibit antiproliferative activity toward a human squamous cell carcinoma cell line, ${ }^{3)}$ to exert antimutagenic activity, ${ }^{4}$ to suppress the induction of matrix metalloproteinase- 9 and prostaglandin $\mathrm{E}_{2}$ release, ${ }^{5)}$ and to suppress the formation of tumor promoter-induced papilloma. ${ }^{6}$ Although several biochemical properties of nobiletin have been extensively studied, the metabolism of this compound has received little attention. ${ }^{7)}$ As nobiletin is a major constituent of our regular diet, especially those rich in citrus fruit flavoring, extracts, or citrus fruit juice, we investigated the in vivo metabolism of this compound. The present paper reports the identification and structure elucidation of the urinary metabolites of nobiletin orally administered to rats.

\footnotetext{
Experimental

Animal Experiments Nobiletin $(200 \mathrm{mg} / \mathrm{kg})$ was administered orally to male Sprague-Dawley (SD) rats and urine specimens were obtained over a 24-h period using a metabolic cage. Methanol $(5.0 \mathrm{ml})$ was added to $1.0 \mathrm{ml}$ of the urine, the mixture was filtered through a $0.45-\mu \mathrm{m}$ filter, and then 20 $\mu \mathrm{l}$ of the sample was injected onto an HPLC column.

HPLC The mobile phase was a linear gradient system comprised of $0.05 \%$ trifluoroacetic acid (solvent A) and 100\% acetonitrile (solvent B), with $\mathrm{A} / \mathrm{B}=95 / 5(0 \mathrm{~min}) \rightarrow 60 / 40(50 \mathrm{~min}) \rightarrow 40 / 60(70 \mathrm{~min})$. The flow rate was $1.0 \mathrm{ml} / \mathrm{min}$ at $40^{\circ} \mathrm{C}$.

Isolation of Metabolites For isolation of urinary metabolites, $3.0 \mathrm{~g}$ of nobiletin was orally administered in portions to each of 12 rats at 200 $\mathrm{mg} / \mathrm{kg} / \mathrm{d}$ over a period of $30 \mathrm{~d}$ and urine samples were collected using metabolic cages. The combined urine samples (1.21) were chromatographed on Sephadex LH-20 with methanol as the eluant. The fractions containing metabolites ( $\mathbf{1}$ and nobiletin, and $\mathbf{2}$ and $\mathbf{3}$ ) were subjected to preparatory HPLC under the following conditions: column, TSK gel ODS-120T $(10 \mu \mathrm{m}$, $300 \times 7.8 \mathrm{~mm}$ i.d., Tosoh Company Ltd., Tokyo, Japan); mobile phase, $20 \%$ methanol (solvent A) and $100 \%$ methanol (solvent B), linear gradient system, $\mathrm{A} / \mathrm{B}=95 / 5(0 \mathrm{~min}) \rightarrow 20 / 80(120 \mathrm{~min})$. The flow rate was $1.0 \mathrm{ml} / \mathrm{min}$ at room temperature. Each metabolite fraction was evaporated to dryness at $40{ }^{\circ} \mathrm{C}$ in vacuo to give $\mathbf{1}(10 \mathrm{mg}), \mathbf{2}(3 \mathrm{mg}), \mathbf{3}(2 \mathrm{mg})$ and nobiletin $(1 \mathrm{mg})$, respectively.
}

\section{Results}

The 3D-HPLC profile of urine samples from rats after oral administration of nobiletin showed one major and two minor distinct peaks tentatively designated as $\mathbf{1}, \mathbf{2}$, and $\mathbf{3}$ in decreasing order of polarity (Fig. 1). Interestingly, little unchanged nobiletin was detected in the urine samples. Metabolites 1, 2, and 3 from urine samples were isolated by chromatographic separation on a Sephadex LH-20 column and repeated preparatory HPLC as described in the experimental section, followed by determination of their chemical structures.

Metabolite 1 was obtained as a yellow powder, mp 139$141^{\circ} \mathrm{C}$. Its molecular formula was $\mathrm{C}_{20} \mathrm{H}_{20} \mathrm{O}_{8}$ based on its high-resolution mass spectrum (HR-MS), which indicated the loss of only a single methyl group. In the UV spectrum of 1, the addition of sodium methoxide to the methanolic solution showed 63-nm bathochromic shifts of band 1 without a decrease in absorbance, which is indicative of a $4^{\prime}$-hydroxyl group. Thus demethylation apparently occurred in position C-4' ${ }^{\prime}$, and 1 was concluded to be $4^{\prime}$-hydroxy-3',5,6,7,8-pentamethoxyflavone. The structure of 1 was also confirmed by a comparison with reference data on $4^{\prime}$-hydroxy-3',5,6,7,8pentamethoxyflavone. ${ }^{8)}$ The minor metabolites $\mathbf{2}$ and $\mathbf{3}$ were not present in sufficient amounts to perform a complete structural elucidation. However, five and four methoxy signals observed in the ${ }^{1} \mathrm{H}-\mathrm{NMR}$ spectrum and the molecular ion peaks found at $\mathrm{m} / \mathrm{z} 388$ and 374 in the electron impact (EI)-MS spectrum, respectively, indicated that these metabolites were the mono- and didemethylated derivatives of nobiletin, respectively. Additionally, as no low-field proton was present in each ${ }^{1} \mathrm{H}-\mathrm{NMR}$ spectrum, the free hydroxyl group was not positioned at $\mathrm{C}-5$, suggesting that demethylation apparently occurred except at the C-5 position.

The urinary amounts of 1 excreted during $24 \mathrm{~h}$ corresponded to $13.19 \pm 1.43 \%$ (mean \pm S.E., $n=3)$ of the dose for nobiletin administration. On the other hand, we could not detect nobiletin per se in the urine after nobiletin administration (detection limited $10 \mathrm{ng} / \mathrm{ml}$, signal $/$ noise $=5$ ).

\section{Discussion}

Although several biochemical properties of nobiletin have been extensively studied, there is information regarding the metabolism of this compound. Additionally, little attention has been directed to the metabolism of polymethoxylflavone. Murakami et al. recently investigated the in vitro metabolism of nobiletin in rat liver microsomes and identified the major metabolites as the $3^{\prime}$-demethylation products of nobiletin. ${ }^{7}$ The identification of nobiletin metabolites in vivo, however, has not previously been performed, and therefore we investigated the metabolism of nobiletin in rats.

The present study on the in vivo metabolic fate of nobiletin focused on the detection and structural elucidation of 
(a)

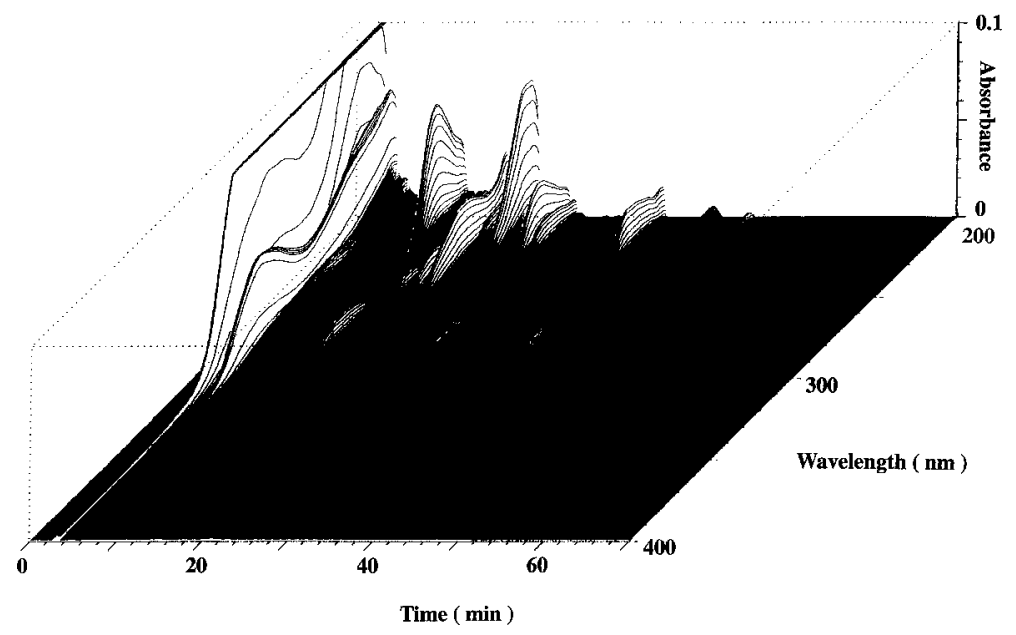

(b)

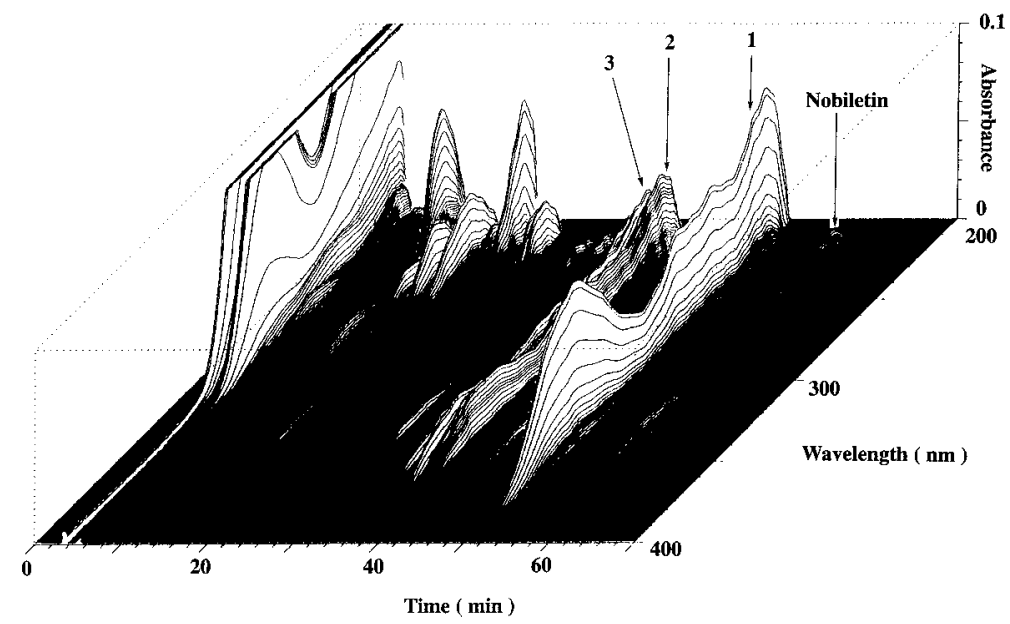

Fig. 1. Three-Dimensional HPLC Chromatograms of Control Urine (a) and Urine Excreted during $24 \mathrm{~h}$ after Oral Administration of Nobiletin (200 mg/kg) (b) to Rats

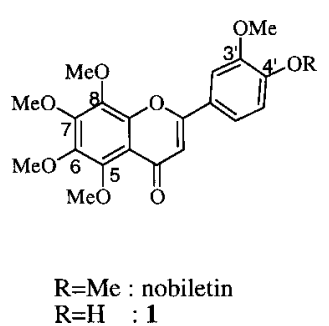

Fig. 2. Structures of Nobiletin and Its Urinary Metabolite (1) in Rats

metabolites with the intact flavonoid structure excreted in rat urine. By using an HPLC column equipped with a photodiode array detector, three metabolites of nobiletin were detected and one major urinary metabolite was determined by spectroscopic methods. To our knowledge, this metabolite has not previously been described as an in vivo metabolite of nobiletin. Although one major metabolite was reported as the 3 '-demethylated product of nobiletin in an in vitro study using rat liver microsomes, this product was not detected in the present study. The nobiletin metabolites identified were all products of the demethylation reaction. The C-4' position appeared to be the primary site for nobiletin demethylation, since the major metabolite was demethylated at the C-4' position. This is in agreement with the results of a previous investigation, where the demethylated products were isolated from rat urine and feces after oral administration of polymethoxyflavone tangeretin. ${ }^{9)}$

Knowledge of the metabolic transformation of nobiletin in vivo is indispensable to evaluate its biological effects fully. It is noteworthy that nobiletin was extensively metabolized to demethylated derivatives, and thereby the nobiletin responsible for the observed biological effects may be the metabolites produced rather than the parent compound. Since some natural compounds become bioactive after being metabolized, further studies are now underway to determine the biological activities of these metabolites.

\section{References}

1) Ben-Aziz A., Science, 155, 1026-1027 (1967).

2) Sugiyama S., Umemura K., Kuroyanagi M., Ueno A., Taki T., Chem. Pharm. Bull., 41, 714-719 (1993).

3) Kandawaswami C., Perkins E., Soloniuk D. S., Drzewiecki G., Middleton E., Jr., Cancer Lett., 56, 147-152 (1991).

4) Wall M. E., Wani M. C., Manikumar G., Abraham P., Taylor H., Hughes T. J., Warner J., McGivney R., J. Nat. Prod., 51, 1084-1091 (1998).

5) Ishiwa J., Sato T., Mimaki Y., Sashida Y., Yano M., Ito A., J. Rheuma- 
tol., 27, 20-25 (2000).

6) Murakami A., Nakamura Y., Torikai K., Tanaka T., Koshiba T., Koshimizu K., Kuwahara S., Takahashi Y., Ogawa K., Yano M., Tokuda H., Nishino H., Mimaki Y., Sashida Y., Kitanaka S., Ohigashi H., Cancer Res., 60, 5059-5066 (2000).

7) Murakami A., Kuwahara S., Takahashi Y., Ito C., Furukawa H., Ju-Ichi
M., Koshimizu K., Ohigashi H., Biosci. Biotechnol. Biochem., 65, 194-197 (2001).

8) Herz W., Kulanthaivel P., Phytochemistry, 21, 2363-2366 (1982).

9) Nielsen S. E., Breinholt V., Cornett C., Dragsted L. O., Food Chem. Toxicol., 38, 739-746 (2000). 